\title{
Clearing the smoke
}

There is an urgent need for more research on the effects of e-cigarettes and nicotine addiction in general.

$\mathbf{E}^{2}$ cigarettes, which allow users to inhale vaporized liquid nicotine rather than the smoke from burning tobacco, have increased in popularity in the US and Europe, with an estimated 2.5 million users in the US alone. Although generally assumed to be safer than conventional cigarettes, e-cigarettes still contain nicotine and some products also contain chemicals that are known carcinogens. Recently proposed FDA regulations would prohibit the sale of newly designed tobacco products to people under 18, would require FDA review before new devices are marketed and would require health warnings on e-cigarette packages; discussions about limits to marketing and adding flavoring to products are ongoing. Although most would likely agree with some of these regulations, such as preventing the sale of e-cigarettes to minors, other aspects of the proposed regulations pose serious questions. Given how little we know about e-cigarettes and their effects on tobacco use, nicotine addiction and long-term health, what would the proposed health warnings say? And if there are to be restrictions on the marketing of e-cigarettes, what would these restrictions be? These proposed regulations highlight how little we understand about the consequences of e-cigarette exposure and use, and how urgent the need is for additional research on e-cigarettes and nicotine addiction in general.

One of the biggest questions surrounding e-cigarettes is whether their use increases or decreases nicotine and tobacco use overall. There is evidence that children and adolescents are using e-cigarettes (the CDC reports that $10 \%$ of high school-aged children used these products in 2012, an increase over the previous year) and that many of these children had never used other tobacco products before trying e-cigarettes (20\% of middle school users of e-cigarettes, according to the CDC). There is copious research documenting the harmful effects of nicotine exposure in children and adolescents; in this respect, the FDA's proposed ban on the sale of e-cigarettes to minors seems to be justified. Whether sale to adults should also be restricted is less clear. It is unknown whether adults who had not previously smoked are beginning to use e-cigarettes, perhaps because they are perceived to be safer or less offensive than cigarettes. Further study of this issue is warranted.

E-cigarettes might seem like a safer alternative, but do they actually help addicts to quit? A handful of studies, both clinical trials and longitudinal studies, have failed to come to a consensus on whether the use of e-cigarettes by those already addicted results in higher rates of abstinence. Although some have found that e-cigarettes are effective in helping smokers to quit, others have shown continued use of both e-cigarettes and cigarettes, suggesting a failure to quit smoking. Resolving this issue is crucial. If e-cigarettes are not an effective way to quit smoking, their marketing and use should be restricted as much as possible. But if e-cigarettes could help those still fighting their addiction, marketing campaigns publicizing their efficacy would be justified. It is imperative that further research address this outstanding question.
One of the reasons previous studies have not been able come to a consensus on the effects of e-cigarette use is that the products themselves are heterogeneous. Nicotine absorption varies among different devices, and may even vary among users of the same device for those devices that allow users to refill nicotine liquid themselves. Although it is clear that different dosing regimens and methods of administration of nicotine can have vastly different effects on people, this is a factor that is not often considered carefully enough in studies in humans or animals. There are of course technical limitations inherent in getting experimental animals to smoke cigarettes, but animal researchers should try to replicate human administration methods as closely as possible, both for nicotine and for other drugs. One major distinction is having an animal self-administer a drug versus having an experimenter administer it; self-administered drugs have been shown to cause changes in the brain and behavior in some cases in which experimenter-administered drugs do not. Beyond this simpler distinction, however, the method of administration of drugs varies widely, and is not always aligned with human consumption habits. For example, some rodent studies involve animals drinking nicotine or inhaling alcohol vapor, neither of which is commonly done by humans. Studies investigating the efficacy of e-cigarettes as smoking cessation aids, for example, should carefully examine different rates of nicotine absorption, as this may profoundly influence results.

These issues of route of administration also apply to toxins or potential carcinogens found in e-cigarettes. For example, one chemical found in most e-cigarettes is propylene glycol, a common constituent of many foods. Although it may be safe to eat, whether it is safe to inhale is unknown. Some e-cigarettes may also contain known carcinogens (such as nitrosamines), and nicotine itself may be a carcinogen (a question that has been hard to decisively answer in the past given all of the other carcinogens present in cigarettes). Further research on the effects of all of the chemicals found in e-cigarettes and their long-term effects on users, as well as those exposed second-hand, is clearly necessary.

As the use of e-cigarettes grows rapidly, it is understandable for the FDA to seek to regulate their marketing, sale and use. The US would not be the first country to do so-the EU is already acting to regulate e-cigarettes, and Brazil and Norway have banned them entirely. However, given our extremely limited knowledge of the effects of these products, both on the rates of nicotine use and abstinence and on general health, much more research is necessary. Despite the fact that tobacco use is the number one preventable cause of morbidity and mortality in the United States, funding for research on nicotine addiction lags behind that for other drugs, such as cocaine. Our lack of understanding of the effects of e-cigarettes highlights this imbalance. More research on the effects of e-cigarettes, and nicotine addiction in general, which will require a stronger commitment from funding agencies, is sorely needed before we can make a truly informed effort to combat nicotine addiction in the US and worldwide. 